\title{
BMJ Open Individual participant data (IPD) meta- analysis of psychological relapse prevention interventions versus control for patients in remission from depression: a protocol
}

Josefien J F Breedvelt (D , ${ }^{1}$ Fiona C Warren, ${ }^{2}$ Marlies E Brouwer, ${ }^{1}$ Eirini Karyotaki, ${ }^{3,4}$ Willem Kuyken (D) , ${ }^{5}$ Pim Cuijpers (D) , ${ }^{3}$ Patricia van Oppen, ${ }^{6}$ Simon Gilbody, Claudi L H Bockting ${ }^{1}$

To cite: Breedvelt JJF, Warren FC, Brouwer ME, et al. Individual participant data (IPD) meta-analysis of psychological relapse prevention interventions versus control for patients in remission from depression: a protocol. BMJ Open 2020;10:e034158. doi:10.1136/ bmjopen-2019-034158

- Prepublication history and additional material for this paper are available online. To view these files, please visit the journal online (http://dx.doi. org/10.1136/bmjopen-2019034158).

Received 13 September 2019 Revised 15 December 2019 Accepted 17 December 2019

Check for updates

(C) Author(s) (or their employer(s)) 2020. Re-use permitted under CC BY. Published by BMJ.

For numbered affiliations see end of article.

Correspondence to Josefien J F Breedvelt; j.j.breedvelt@amsterdamumc.nl

\section{ABSTRACT}

Introduction Psychological interventions and antidepressant medication can be effective interventions to prevent depressive relapse for patients currently in remission of depression. Less is known about overall factors that predict or moderate treatment response for patients receiving a psychological intervention for recurrent depression. This is a protocol for an individual participant data (IPD) meta-analysis which aims to assess predictors and moderators of relapse or recurrence for patients currently in remission from depression. Methods and analysis Searches of PubMed, PsycINFO, Embase and Cochrane Central Register of Controlled Trials were completed on 13 0ctober 2019. Study extractions and risk of bias assessments have been completed. Study authors will be asked to contribute IPD. Standard aggregate meta-analysis and IPD analysis will be conducted, and the outcomes will be compared with assess whether results differ between studies supplying data and those that did not. IPD files of individual data will be merged and variables homogenised where possible for consistency. IPD will be analysed via Cox regression and one and two-stage analyses will be conducted.

Ethics and dissemination The results will be published in peer review journals and shared in a policy briefing as well as accessible formats and shared with a range of stakeholders. The results will inform patients and clinicians and researchers about our current understanding of more personalised ways to prevent a depressive relapse. No local ethics approval was necessary following consultation with the legal department. Guidance on patient data storage and management will be adhered to.

PROSPERO registration number CRD42019127844.

\section{INTRODUCTION}

Depression is a highly debilitating mental health problem, and one of the leading causes of disability worldwide. ${ }^{1}$ Depression or major depressive disorder (MDD) has a chronic course, and relapse rates are high. ${ }^{23}$ Approximately $40 \%-60 \%$ of patients who develop a

\section{Strengths and limitations of this study}

- An individual participant data (IPD) analysis can be a superior method to standard meta-analyses, as it allows us to increase power to detect potential predictors and moderators of treatment.

- This is the first IPD meta-analysis which sets out to evaluate a wide range of psychological relapse prevention interventions for depression.

- This, to our knowledge, is the largest IPD metaanalysis thus far to assess the effects of moderators on relapse and recurrence of depression.

- IPD meta-analyses are limited by the data available, which may limit the number of studies included and subsequently reduce statistical power and limit generalisability.

- Moreover, there may be inconsistencies in terms of how covariates are reported, limiting the number and range of moderators that can be included in the analysis.

major depressive episode relapse, and this risk increases up to $90 \%$ after three episodes. ${ }^{4-7}$

Recurrence and relapse are terms frequently used synonymously to describe the reoccurrence of depressive symptoms. ${ }^{89}$ Both terms indicate a worsening of symptoms and onset of a new episode of depression after a period of no or subthreshold symptomatology. However, they do so at different time points after the initial episode. ${ }^{89}$ The clinical and scientific distinctions on these time cutoffs between relapse and recurrence were found to be unhelpful. ${ }^{10}$ Therefore we will use relapse to describe both recurrence and relapse in this manuscript.

Two types of interventions are recommended for relapse prevention for remitted or recovered MDD patients; either continuation 
of antidepressant medication (ADM) or psychological interventions. ${ }^{11} 12 \mathrm{ADM}$ or psychological interventions can be offered as a continuation following the therapy on which remission was achieved (continuation therapy), or as sequential interventions, where a different therapy is offered after (spontaneous) remission is achieved. ${ }^{41314}$ Mindfulness-based cognitive therapy (MBCT), preventive cognitive therapy (PCT) and wellbeing therapy (WBT) are examples of psychological relapse prevention interventions used in sequential therapy. ${ }^{24} 1315-19$

A substantial proportion of patients still relapse even after having received a psychological intervention, ADM or a combination of these after remission to prevent relapse. ${ }^{19}{ }^{20}$ It might be that some psychological intervention types might be more effective, or they have varying levels of effectiveness for different patient characteristics. These characteristics can be separated into prognostic indicators and prescriptive factors. ${ }^{21}$ Prognostic factors affect the course of depression regardless of the treatment provided. ${ }^{21}$ Prescriptive factors, also called 'moderators', indicate differential treatment effects across patient characteristics and can be used inform decisions as to which treatment may be best suited to someone with specific characteristics, such as continuation or sequential treatment and/or indicated treatment approaches. ${ }^{21} 22$

A recent meta-analytic review of reviews found that, in recurrent depression, childhood maltreatment, previous depressive episodes, residual symptoms after treatment, co-occurring anxiety disorders as well as rumination were among the strongest prognostic factors in recurrent depression. ${ }^{23}$ In terms of moderators, there was less evidence to suggest any variable could affect treatment selection. Some evidence suggests that residual symptoms at baseline may be potentially be reduced more by cognitive behavioural therapy (CBT) compared with ADM and patients with a history of severe childhood maltreatment may respond better to MBCT compared with treatment as usual (TAU) ${ }^{23}$ However, it remains unclear whether treatment outcomes can be improved if certain interventions are offered to patients with these characteristics. ${ }^{423} 24$

An individual participant data (IPD) meta-analysis can provide a unique insight into treatment effectiveness and the effect of individual-level moderators on treatment outcome. ${ }^{25}{ }^{26}$ IPD analyses are relatively new in the field of clinical psychology and psychiatry. ${ }^{27} 28$ They can provide a more accurate estimate of the true treatment effect and help identify which interventions work better or worse in specific subgroups of patients. ${ }^{26}$ The use of IPD from multiple studies combined can increase the power to detect which patients respond best to treatment within and across studies, something which cannot be assessed with aggregate trial information alone. ${ }^{29}$ Besides, an IPD meta-analysis can adjust for study-level confounding factors which may bias the result of a traditional aggregate meta-analysis. ${ }^{30}$

To our knowledge, only one IPD meta-analysis evaluating psychological interventions versus control for recurrent depression has been conducted thus far. ${ }^{31}$ In this IPD meta-analysis, Kuyken and colleagues estimated the effectiveness of MBCT versus control (active or non-active) and aimed to establish the effect of moderators on treatment outcome. Among nine included studies with 1258 participants, the authors performed a time to event (depressive relapse) IPD meta-analysis which produced an HR of 0.69 (95\% CI 0.58 to 0.82 ), indicating a reduced risk of relapse in those participants who received MBCT compared with participants in the control group. No differential treatment effects across participant characteristics were found. In this review, depression baseline scores (indicative of incomplete recovery) predicted a stronger effect at follow-up. Thus, patients with higher symptomatology at the start of treatment seemed to benefit most. Kuyken et $a l^{31}$ only included MBCT, a range of other forms of psychological interventions were not included (ie, CBT, PCT or interpersonal therapy). Including these would increase power to assess moderators of treatment, establish effects of different psychological interventions versus control, as well as allow for an evaluation of the interaction between therapy type and moderator.

This study aims to answer three research questions which remain pertinent from the literature, namely:

1. What are the effects of different preventive psychological interventions on reducing the risk of relapse versus control?

2. What are the predictor variables associated with an increased risk of relapse for patients receiving a psychological intervention?

3. What factors (moderators) may predict which participant responds best to which type of intervention?

\section{METHODS}

\section{General approach}

This IPD meta-analysis is registered on PROSPERO and any key changes or amendments will be documented there. The protocol is registered retrospectively as searches and aggregate study-level extractions were already conducted in 2018, and the last search was conducted on 13 October 2019. The Preferred Reporting Items for Systematic Reviews and Meta-Analyses IPD statement will be followed for the reporting of this study. ${ }^{32}$

\section{Systematic review to identify eligible papers \\ Eligibility criteria \\ Types of studies}

Eligible studies must have a randomised controlled study (RCT) design and be written in the English language.

\section{Types of interventions}

Included studies will examine the effects of psychological relapse prevention interventions, and we used the following definition: 'a modality of treatment in which the therapist and patient(s) work together to ameliorate psychopathological conditions and functional impairment through focus on the therapeutic relationship; the patient's attitudes, thoughts, affect and behaviour; and social context and development'. ${ }^{33}$ The intervention 
can be delivered in any modality or setting, such as face to face, in a group format or online. Examples include MBCT, PCT, continuation cognitive therapy, or WBT. ${ }^{1314171934}$ Sequential treatment combinations, that is, studies where a different relapse prevention therapy starts directly after treatment for acute depression, ${ }^{13} 14$ will be included as long as patients were randomised to a relapse prevention intervention after reaching remission or recovery (as defined by the study authors) from the acute episode. As we are interested in the overall effect of psychological interventions versus control, studies where participants were randomised to taper from $\mathrm{ADM}$ as they receive the psychological intervention will be excluded.

\section{Types of comparators}

To answer our first question (estimate of treatment effect), at least one non-psychotherapeutic control group should be available. Eligible control conditions include TAU, ADM and active psychological control group. For our second question (prognostic indicators) for patients receiving psychological interventions, any control group can be included. For our third question (moderator analysis), which assesses what works for whom, at least one non-psychological control group should be available.

\section{Types of participants}

Participants aged 18 and over were included. Studies, where participants were only included if they had an onset after the age of 65 , were excluded. As also specified by Brouwer (2019), the factors causing and contributing to the first onset of depression in the 65 and above age range may differ from lower age groups. ${ }^{35-37}$ Participants in trials need to have had at least one prior diagnosis of MDD established by an independent clinical interview and/or healthcare provider who was not involved in the study. The randomisation and subsequent treatment or TAU should have been during the remission, response or recovery phase. Participants were required to be in remission from at least one episode of MDD. Remission is classified as a period of at least 8 weeks, where participants had no or subthreshold clinical symptoms. ${ }^{48}$ Sequential treatment combinations were included as long as participants in the intervention group achieved remission or response according to the authors of the study. Studies were excluded when participants were in treatment for another mental disorder as classified by the Diagnostic and Statistical Manual of Mental Disorders (DSM)-V, DSM-IV, or DSM-III criteria. ${ }^{39} 4041$

\section{Outcomes}

\section{Primary outcome}

The primary outcome of the study will be time to depressive relapse at any point of follow-up measured in weeks. Depressive relapse needs to be determined via an independent examiner/assessor via a diagnostic interview, for example, the Structured Clinical Interview for DSM-IV Axis 1 Disorders, ${ }^{42}$ the Mini-International Neuropsychiatric
Interview, ${ }^{43} 44$ the Composite International Diagnostic Interview $^{45}$ or the Hamilton Depression Rating Scale. ${ }^{46}$

\section{Covariates}

We are interested in patient characteristics which predict relapse of depression regardless of treatment allocation (predictor variables), and those that may affect outcome based on treatment allocation (moderators). Sometimes these terms are used interchangeably. However, in this manuscript we use these terms to differentiate between overall treatment outcome predictors and factors moderating treatment response (ie, who responds better to one treatment vs a control condition or vice versa). Patient characteristics will be included in the analyses if they are consistently reported and available across datasets and justify inclusion based on prior literature that identifies them as potential predictors or moderators. ${ }^{423} 47$

We will ask the individual studies to provide data on the following set of variables which could either be predictors or moderators of effect.

Age at baseline, gender, ethnicity, country of birth, education status, employment status, marital status, number of previous depressive episodes, age of onset of first episode of depression, time spent in remission since last episode, duration of past episode of depression, stable or unstable remission since last episode, history of childhood trauma, previously received psychotherapy for MDD (including type and time since last session), previously received medication for MDD (including type, dosage and time since last intake), comorbid anxiety disorder, comorbid mental health disorder, comorbid physical health disorder, antidepressant exposure at baseline, baseline depression symptoms, baseline anxiety symptoms, baseline quality of life symptoms.

\section{Timing of outcome measures}

The primary outcome, time to depressive relapse, will be included for all participants, regardless of variation in follow-up duration across studies. We will consider censoring follow-up time, for example at 60 weeks, contingent on the length of follow-up across studies.

\section{Secondary outcomes}

Secondary outcomes include depressive symptomatology at follow-up, anxiety symptoms and quality of life. These will be collected and may be used for future analysis.

\section{Searches for study identification and selection}

PubMed, PsychINFO, Embase and Cochrane Central Register of Controlled Trials were searched. Index and free terms, jointly with Boolean operators, were used on four tiers, namely: (1) depressive disorder, (2) recurrence and relapse, (3) preventative interventions, (4) RCT. Online supplementary appendix 1 shows the search terms for PubMed. References from previous meta-analyses were screened to ensure no RCTs are missing. ${ }^{1419-51}$ Key authors in the area of relapse prevention for depression were consulted for additional literature and unpublished manuscripts. 
The last searches for this IPD were completed on 13 October 2019, three researchers independently screened search results (JB screening all and a research assistant or collaborator conducting the second screen). Full-text screens were conducted by two independent researchers. Outcome data for all studies are being extracted independently by at least two independent researchers and are then being merged and checked by JB. Discrepancies were discussed with the researchers involved in extraction and resolved via discussion with MB.

\section{Quality assessment}

Study quality will be assessed by two reviewers who will independently evaluate the studies based on six criteria for risk of bias from the Risk of Bias tool by the Cochrane Collaboration. ${ }^{5253}$ The following criteria will be assessed: (1) random sequence generation; (2) allocation concealment; (3) blinding of outcome assessors; (4) incomplete outcome data; (5) selective outcome reporting; (6) other threats to validity (similar groups, cointerventions, compliance and similar timing of outcome assessment). Studies will be rated on each criterion with either 'low risk' 'high risk' 'unclear risk'. A minimum of five criteria with a 'low risk' rating qualified as the overall low risk of bias. Only data that are published in the full-text paper of the trial will be evaluated and assessed by two independent assessors, to avoid potential imbalance between studies that can share data and those that are not able to do so.

\section{Patient and public involvement}

During the development of this protocol, no direct patient involvement has taken place. However, we are planning to consult with people with lived experience during the interpretation and dissemination of the study results. Results will be disseminated in peer review journals but also a coproduced evidence briefing in collaboration with the Mental Health Foundation. We have set up a consortium of all collaborating authors on this IPD (and other researchers as well as other stakeholders interested in relapse prevention of depression) titled "International Taskforce For RelApse prevention of depression (ITFRA)". This taskforce aims to help disseminate the research and raise awareness with the public and clinical community.

\section{IPD data collection and aggregation \\ Invitation of authors}

All corresponding authors of the selected articles for inclusion will be contacted via email by the senior authors of this article with an invitation to participate in the study. The letter includes details regarding the study group, the study proposal, the goals of the analysis, the variables of interest and includes a request to share raw data from the trial participants for this study. When there is no response from the author within 4 weeks, we will try a second time. If this fails, a second senior author will be contacted. We will continue until we have reached at least three authors. If none of the authors responds or if all the authors indicate that the data are unavailable or that they are unable to share due to access restrictions, it will be noted that the study data were unavailable. We will send a maximum of four reminders until we exclude the study as being unavailable.

\section{Data checking and integrity}

After accepting the invitation to collaborate and signing the data transfer agreement, the authors will be asked to share their data via a secure data transfer portal available to the Amsterdam University Medical Centre-Location AMC. The received data will be reviewed to assess the completeness and accuracy of the dataset. If any inconsistencies are present (missing data, inconsistencies or extreme values, discrepancies between the trial report and the data), the issue will be discussed with the study authors who will be contacted for clarification. The study progress and discrepancies will be recorded.

\section{Creating a database and aggregation}

A template spreadsheet with study characteristics and outcome data will be created. Once data have been checked and standardised, it will be merged into the final file for analysis. All individual datasets will be merged into one large IPD dataset. Once all data have been merged into a final dataset, it will be rechecked for accuracy by a researcher in the study team, by comparing participant numbers, descriptive data and relapse/recurrence data to the reported data in the peer-review article.

\section{Statistical analysis}

To determine the final selection of potential predictors and moderators, we will identify reported covariates across studies and identify ways that covariates can be appropriately standardised across studies, for example, by collapsing categories. Baseline covariates will be included in the modelling if the covariate has at least $40 \%$ available data (ie, non-missing) in at least three studies. ${ }^{54}$

To examine the overall effects of the interventions compared with control (research question 1) and assess the modifying effect of study and individual-level variables on prognostic and predictive value (research questions 2 and 3), we will conduct one-stage and two-stage randomeffects IPD meta-analyses using Stata v. $14 .^{55}$

Our primary meta-analysis method will be the one-stage random-effects approach; we will seek to perform onestage random-effects meta-analyses for the time to event outcomes using hierarchical flexible parametric models. ${ }^{56}$ A random-effects meta-analysis was chosen because we are aware of clinical heterogeneity in the included trials (eg, due to differences in study populations, types of psychotherapy, or differences in the control group), which may result in statistical heterogeneity. Should such models fail to converge, we will then perform Cox proportional hazards models stratified by study (one-stage fixed effect approach).

A two-stage method for time to event data calculates the HR for relapse for each study individually, using a Cox proportional hazards model; these HRs will then be 
combined in the IPD meta-analysis. If only rate ratio data are available, we aim to calculate an estimate of HR data from rate ratio data by the logarithms of event-free proportions. ${ }^{57}$ We will use the DerSimonian and Laird ${ }^{58}$ random effects method to combine the results of the individual studies and will apply the Hartung Knapp-Sidik-Jonkman method correction to account for uncertainty in $\tau^{2} .5960$

We aim to answer our first research question (estimate of effect) via an IPD meta-analysis on a maximum of four pairwise comparisons: (1) psychotherapy versus ADM, (2) psychotherapy versus TAU, (3) psychotherapy versus active control (ie, placebo or active psychological intervention), (4) psychotherapy versus any nonpsychotherapy control. All analyses will use the intention to treat approach, whereby all participants will be included in the analyses according to their randomised allocation irrespective of treatment received. Primary analyses will use clinical and demographic participant baseline characteristics and time to event outcome data.

To assess statistical heterogeneity, we will assess $\mathrm{I}^{226}$ derived from two-stage meta-analysis models, with $0 \%$ indicating no heterogeneity, $25 \%$ low heterogeneity, $50 \%$ moderate heterogeneity and $75 \%$ as being high heterogeneity. ${ }^{26}$ A $95 \%$ prediction interval will be calculated to evaluate the potential range of the treatment effect when applied in an individual study setting. ${ }^{28}$

\section{Missing data}

The percentage of individual participant missing data (baseline characteristics, event status and time to event or censoring) will be recorded for each study. We plan to use multiple imputation on participant baseline characteristics which are missing individually within studies reporting the specified characteristic ${ }^{61}$ and a sensitivity analysis would be performed using observed and imputed data.

\section{Sensitivity analysis}

To assess whether various sources of heterogeneity affect the overall effect size and the robustness of the IPD findings, we will conduct sensitivity analyses. Sources of heterogeneity, including study level characteristics such as the risk of bias, year of publication, setting (community, primary or secondary care), duration of follow-up, country of study, will be explored. We will also explore differences between one and two-stage IPD meta-analytic approaches. ${ }^{62}$

\section{Aggregate data meta-analysis}

We will investigate the possibility of inclusion bias by reporting the characteristics of eligible trials for which data were sought but not obtained. If suitable results are available for these studies, we will perform two-stage meta-analyses to incorporate the results of these trials with those of the trials where IPD was obtained. Potential publication bias will be estimated by funnel plot inspection and by use of Egger's test for asymmetry. ${ }^{63}$

\section{Predictors and moderators}

To investigate the effects of baseline participant characteristics on relapse regardless of the psychological intervention offered (research question 2), each of the predictors will be entered into a time-to-event model with the predictor as the independent variable (together with treatment allocation) and time to relapse as the dependent variable. To account for clustering of patients within a study we will include a study as a variable within the model, either as a random or fixed effect. Individual predictor $v$ will be selected based on $p$ values. We will initially include variables that are associated with the outcome with $\mathrm{p}<0.10$, in regression models, including an individual variable and treatment allocation only. These variables will then be combined in a further model; any variables with $\mathrm{p}>0.05$ will then be removed, which follows the criteria by Heffner $e t a l^{64}$ as described in Ahmed et al. ${ }^{65}$ Each variable that has been removed will then be added individually to the model where all variables have $\mathrm{p}<0.05$ and will be included if their $\mathrm{p}$ value is $<0.05$. In this way, predictors of time to depressive relapse will be identified. We will also assess the model fit by Akaike's Information Criterion. ${ }^{66}$

To investigate differential treatment effects across different participant subgroups (research question 3), we will perform a series of models adding the interaction term between each moderator and treatment allocation (each model will include only one interaction effect). In the two-stage model, we will first estimate the interaction at study level and will then combine interaction estimates in a random effects-meta-analysis. Potential moderators will be centred within the study to avoid ecological bias; continuous covariates will be centred around the study mean; binary covariates will be centred around the proportion with the characteristic. ${ }^{28} 67$ Treatment allocation for the above model will be categorised by psychological intervention versus non-psychological intervention in the first instance.

\section{DISCUSSION}

This IPD meta-analysis can provide up to date treatment efficacy estimates and has the potential to establish whether there are any moderators and predictors of treatment effect. This could help answer the 'what works for whom' question we are hoping to answer. The IPD has several strengths, first it allows us to look at individual-level data rather than studylevel data which give us the possibility to assess whether specific baseline and treatment characteristics predict or moderate time to relapse with more specificity and more power. Moreover, we will be able to study our outcome of interest more precisely, using the individual time to event data rather than aggregate information presented at study level. ${ }^{68}$ Various factors may hamper the process and interpretation of an IPD meta-analysis. There may be inconsistencies across studies regarding which variables are reported, and how they are reported, thus limiting our ability to assess the effect of these potential predictors and moderators of treatment. There may be some inclusion bias, as we may not be able to obtain IPD for all eligible studies; however, where possible, sensitivity analyses with the inclusion of published aggregate data will address this issue. 
This is to our knowledge the first IPD meta-analysis that attempts to establish the effects of a range of psychological relapse prevention strategies compared with control conditions, as well as assessing whether any predictors or moderators might affect the risk of relapse for patients receiving a psychological intervention or a control intervention.

This study has the potential to inform clinicians, healthcare providers and people who have had a previous episode of depression. Firstly on the relative effectiveness of different approaches for prevention of depressive relapse and secondly this study may also offer further indications on overall risk factors and what may work for whom in preventing relapse for patients who have experienced a previous episode of depression.

\section{Ethics and dissemination}

We will disseminate the work via peer review publications. Day to day oversight and management of the database will be by JB and analyses will be conducted by JB and FCW. The dataset is not open access. Researchers may potentially receive access to the database on request pending on institutional approvals on data transfer and ethics and approval of all the collaborating co-authors for sharing the data beyond the ITFRA consortium. Patient privacy will be ensured by adhering to the Amsterdam University Medical Centre-location AMC guidance on research participant data management and storage. This covers a range of data protection and storage measures including (1) setting up inter-institutional data sharing agreements prior to data sharing, (2) sharing only pseudonymised (de-identified) data, (3) secure data sharing and data storage.

\section{Author affiliations}

${ }^{1}$ Department of Psychiatry and Amsterdam Public Health research institute, Amsterdam University Medical Centre - Location AMC, Amsterdam, The Netherlands ${ }^{2}$ Institute of Health Research, College of Medicine \& Health, University of Exeter, Exeter, UK

${ }^{3}$ Department of Clinical, Neuro and Developmental Psychology, Amsterdam Public Health research institute, Vrije Universiteit Amsterdam, Amsterdam, The Netherlands ${ }^{4}$ Department of Global Health and Social Medicine, Harvard Medical School, Boston, Massachusetts, USA

${ }^{5}$ Department of Psychiatry, University of Oxford, Oxford, UK

${ }^{6}$ Department of Psychiatry, Amsterdam Public Health research institute, Amsterdam University Medical Centre, location VUmc and GGZ InGeest, Amsterdam, Netherlands ${ }^{7}$ Mental Health and Addictions Research Group - Department of Health Sciences, The University of York, York, UK

Correction notice This article has been corrected since it was published. The names of the authors,Fiona C Warren, Marlies E Brouwer, and Patricia van Oppen, have been corrected.

Twitter Josefien J F Breedvelt @josefienUMH, Eirini Karyotaki @KaryotakiEirini, Willem Kuyken @WillemKuyken and Simon Gilbody @SimonGilbody

Acknowledgements We acknowledge Alicia Segovia-Nunez and Emma Boers for supporting with the screening of the studies for inclusion as part of this IPD and we thank Emma Boers for critically reviewing the manuscript.

Contributors JB, MB and CLB conceptualised the study. FCW and WK delivered input on study conceptualisation and FCW specifically, critically revised and reviewed the protocol and statistical analysis plan. EK and PC critically reviewed the protocol for missing intellectual content and accuracy. Pv0 evaluated the study concept and outline, critically reviewed the protocol, all study materials and advised on the consortium. SG critically reviewed the protocol for missing intellectual content and accuracy and provided input on methodological and data access queries.

Funding This work was supported by the Amsterdam Public Health Institute Collaborative grant 2018.

Competing interests CLB is coeditor of PLOS One and receives no honorarium for this role. CLB is also codeveloper of the Dutch multidisciplinary clinical guideline for anxiety and depression, for which she receives no remuneration. She is also a member of the scientific advisory board of the National Insure Institute, for which she receives an honorarium, although this role has no direct relation to this study. CLB has presented keynote addresses at conferences, such as the European Psychiatry Association and the European Conference Association, for which she sometimes receives an honorarium. She has presented clinical training workshops, some of which include a fee. CLB receives royalties from her books and coedited books, and she developed PCT on the basis of the cognitive model of A T Beck. JB is employed by the Mental Health Foundation, a charity focused on promoting and improving mental health.

Patient consent for publication Not required.

Ethics approval Ethical approval was not deemed necessary after consultation with the legal department.

Provenance and peer review Not commissioned; externally peer reviewed.

Open access This is an open access article distributed in accordance with the Creative Commons Attribution 4.0 Unported (CC BY 4.0) license, which permits others to copy, redistribute, remix, transform and build upon this work for any purpose, provided the original work is properly cited, a link to the licence is given, and indication of whether changes were made. See: https://creativecommons.org/ licenses/by/4.0/.

\section{ORCID iDs}

Josefien J F Breedvelt http://orcid.org/0000-0003-1864-1861

Willem Kuyken http://orcid.org/0000-0002-8596-5252

Pim Cuijpers http://orcid.org/0000-0001-5497-2743

\section{REFERENCES}

1 World Health Organisation. Depression and other common mental disorders global health estimates. Geneva, Switzerland, 2017.

2 Beshai S, Dobson KS, Bockting CLH, et al. Relapse and recurrence prevention in depression: current research and future prospects. Clin Psychol Rev 2011;31:1349-60.

3 lancu SC, Wong YM, Rhebergen D, et al. Long-term disability in major depressive disorder: a 6-year follow-up study. Psychol Med 2019;9:1-9.

4 Bockting CL, Hollon SD, Jarrett RB, et al. A lifetime approach to major depressive disorder: the contributions of psychological interventions in preventing relapse and recurrence. Clin Psychol Rev 2015;41:16-26.

5 Eaton WW, Shao H, Nestadt G, et al. Population-based study of first onset and chronicity in major depressive disorder. Arch Gen Psychiatry 2008;65:513-20.

6 Moffitt TE, Caspi A, Taylor A, et al. How common are common mental disorders? Evidence that lifetime prevalence rates are doubled by prospective versus retrospective ascertainment. Psychol Med 2010;40:899-909.

7 Solomon DA, Keller MB, Leon AC, et al. Multiple recurrences of major depressive disorder. Am J Psychiatry 2000;157:229-33.

8 Frank E, Kupfer DJ, Perel JM, et al. Three-Year outcomes for maintenance therapies in recurrent depression. Arch Gen Psychiatry 1990;47:1093-9.

9 Bockting CLH, Smid NH, Koeter MWJ, et al. Enduring effects of preventive cognitive therapy in adults remitted from recurrent depression: a 10 year follow-up of a randomized controlled trial. J Affect Disord 2015;185:188-94.

10 de Zwart PL, Jeronimus BF, de Jonge P. Empirical evidence for definitions of episode, remission, recovery, relapse and recurrence in depression: a systematic review. Epidemiol Psychiatr Sci 2019;28:544-62.

11 American Psychiatric Association [APA]. Practice guidelines for the treatment of patients with major depressive disorder. 3rd edn, 2010.

12 National Institute for Health and Care Excellence. NICE clinical guideline 90. Depression in adults. the treatment and management of depression in adults, 2009. Available: http://www.nice.org.uk/ nicemedia/live/12329/45888/45888.pdf 
13 Guidi J, Tomba E, Fava GA. The sequential integration of pharmacotherapy and psychotherapy in the treatment of major depressive disorder: a meta-analysis of the sequential model and a critical review of the literature. Am J Psychiatry 2016;173:128-37.

14 Guidi J, Fava GA, Fava M, et al. Efficacy of the sequential integration of psychotherapy and pharmacotherapy in major depressive disorder: a preliminary meta-analysis. Psychol Med 2011;41:321-31.

15 Fava GA, Rafanelli C, Cazzaro M, et al. Well-Being therapy. A novel psychotherapeutic approach for residual symptoms of affective disorders. Psychol Med 1998;28:475-80.

16 Kuyken W, Hayes R, Barrett B, et al. Effectiveness and costeffectiveness of mindfulness-based cognitive therapy compared with maintenance antidepressant treatment in the prevention of depressive relapse or recurrence (prevent): a randomised controlled trial. Lancet 2015;386:63-73.

17 Piet J, Hougaard E. The effect of mindfulness-based cognitive therapy for prevention of relapse in recurrent major depressive disorder: a systematic review and meta-analysis. Clin Psychol Rev 2011;31:1032-40.

18 Vittengl JR, Clark LA, Thase ME, et al. Stable remission and recovery after acute-phase cognitive therapy for recurrent major depressive disorder. J Consult Clin Psychol 2014;82:1049-59.

19 Biesheuvel-Leliefeld KEM, Kok GD, Bockting CLH, et al. Effectiveness of psychological interventions in preventing recurrence of depressive disorder: meta-analysis and meta-regression. J Affect Disord 2015;174:400-10.

20 Hughes S, Cohen D. A systematic review of long-term studies of drug treated and non-drug treated depression. J Affect Disord 2009;118:9-18.

21 Fournier JC, DeRubeis RJ, Shelton RC, et al. Prediction of response to medication and cognitive therapy in the treatment of moderate to severe depression. J Consult Clin Psychol 2009;77:775-87.

22 Kraemer HC, Wilson GT, Fairburn CG, et al. Mediators and moderators of treatment effects in randomized clinical trials. Arch Gen Psychiatry 2002;59:877-83.

23 Buckman JEJ, Underwood A, Clarke K, et al. Risk factors for relapse and recurrence of depression in adults and how they operate: a four-phase systematic review and meta-synthesis. Clin Psychol Rev 2018;64:13-38.

24 Holmes EA, Ghaderi A, Harmer CJ, et al. The Lancet psychiatry Commission on psychological treatments research in tomorrow's science. Lancet Psychiatry 2018;5:237-86.

25 Riley RD, Steyerberg EW. Meta-Analysis of a binary outcome using individual participant data and aggregate data. Res Synth Methods 2010;1:2-19.

26 Higgins JPTet al. Measuring inconsistency in meta-analyses. BMJ 2003;327:557-60.

27 Weitz E, Kleiboer A, van Straten A, et al. Individual patient data meta-analysis of combined treatments versus psychotherapy (with or without pill placebo), pharmacotherapy or pill placebo for adult depression: a protocol. BMJ Open 2017;7:e013478-7.

28 Riley RD, Lambert PC, Abo-Zaid G. Meta-Analysis of individual participant data: rationale, conduct, and reporting. $B M J$ 2010;340:c221-5.

29 Lambert PC, Sutton AJ, Abrams KR, et al. A comparison of summary patient-level covariates in meta-regression with individual patient data meta-analysis. J Clin Epidemiol 2002;55:86-94.

30 Berlin JA, Santanna J, Schmid CH, et al. Individual patient- versus group-level data meta-regressions for the investigation of treatment effect modifiers: ecological bias rears its ugly head. Stat Med 2002;21:371-87.

31 Kuyken W, Warren FC, Taylor RS, et al. Efficacy of MindfulnessBased cognitive therapy in prevention of depressive relapse: an individual patient data meta-analysis from randomized trials. JAMA Psychiatry 2016;73:565-74.

32 Stewart LA, Clarke M, Rovers M, et al. Preferred reporting items for systematic review and meta-analyses of individual participant data: the PRISMA-IPD statement. JAMA 2015;313:1657-65.

33 Brent DA, Kolko DJ. Psychotherapy: definitions, mechanisms of action and relationship to etiological models. J Abnorm Child Psychol 1998;26:17-25.

34 Vittengl JR, Clark LA, Dunn TW, et al. Reducing relapse and recurrence in unipolar depression: a comparative meta-analysis of cognitive-behavioral therapy's effects. J Consult Clin Psychol 2007;75:475-88.

38 Brouwer ME. Exploring transgenerational approaches to prevent common mental disorders. Utrecht, the Netherlands: University of Utrecht, 2019.

36 Korten NCM, Comijs HC, Lamers F, et al. Early and late onset depression in young and middle aged adults: differential symptomatology, characteristics and risk factors? J Affect Disord 2012;138:259-67.

37 Devanand DP, Adorno E, Cheng J, et al. Late onset dysthymic disorder and major depression differ from early onset dysthymic disorder and major depression in elderly outpatients. J Affect Disord 2004;78:259-67.

38 Frank E, Prien RF, Jarrett RB, et al. Conceptualization and rationale for consensus definitions of terms in major depressive disorder. remission, recovery, relapse, and recurrence. Arch Gen Psychiatry 1991;48:851.

42 American Psychiatric Association. Diagnostic and statistical manual of mental disorders, American psychiatric association. diagnostic and statistical manual of mental disorders (4th ED). American Psychiatric Publishing, Inc., 1994.

40 Rutter M, Shaffer D. DSM-III. J Am Acad Child Psychiatry 1980;19:371-94.

41 American Psychiatric Association. Diagnostic and statistical manual of mental disorders (5th ED. Arlington, VA: American Psychiatric Association, 2013

42 First MB. Structured clinical interview for the DSM (SCID). Encycl Clin Psychol 2015:1-6.

43 Amorim P. Mini international neuropsychiatric interview (mini). Rev Bras Psiquiatr 2002;22:106-15.

44 Lecrubier Y, Sheehan DV, Weiller E, et al. The mini international neuropsychiatric interview (mini). A short diagnostic structured interview: reliability and validity according to the CIDI. Eur Psyc 1997:12:224-31.

45 Kessler RC, Duncan GJ, Gennetian LA, et al. Associations of housing mobility interventions for children in high-poverty neighborhoods with subsequent mental disorders during adolescence. JAMA 2014;311:937-47.

46 Hamilton M. A rating scale for depression. J Neurol Neurosurg Psychiatry 1960;23:56-62.

47 Burcusa SL, lacono WG. Risk for recurrence in depression. Clin Psychol Rev 2007;27:959-85.

48 Clarke K, Mayo-Wilson E, Kenny J, et al. Can non-pharmacological interventions prevent relapse in adults who have recovered from depression? A systematic review and meta-analysis of randomised controlled trials. Clin Psychol Rev 2015;39:58-70.

49 Klein JP, Berger T, Schröder J, et al. Effects of a psychological Internet intervention in the treatment of mild to moderate depressive symptoms: results of the evident study, a randomized controlled trial. Psychother Psychosom 2016;85:218-28.

50 Rodgers M, Asaria M, Walker S, et al. The clinical effectiveness and cost-effectiveness of low-intensity psychological interventions for the secondary prevention of relapse after depression: a systematic review. Health Technol Assess 2012;16:1-130.

51 Zhang Z, Zhang L, Zhang G, et al. The effect of CBT and its modifications for relapse prevention in major depressive disorder: a systematic review and meta-analysis. BMC Psychiatry 2018;18:50.

52 Furlan AD, Pennick V, Bombardier C, et al. 2009 updated method guidelines for systematic reviews in the Cochrane back review group. Spine 2009;34:1929-41.

53 Higgins JPT, Green S. Cochrane Handbook for systematic reviews of interventions, 2011.

54 Crawford F, Cezard G, Chappell FM, et al. A systematic review and individual patient data meta-analysis of prognostic factors for foot ulceration in people with diabetes: the International research collaboration for the prediction of diabetic foot ulcerations (PODUS). Health Technol Assess 2015:19:1-210.

55 StataCorp (2015). Stata statistical software: release 14. College Station: StataCorp LP, 2015

56 Crowther MJ, Look MP, Riley RD. Multilevel mixed effects parametric survival models using adaptive Gauss-Hermite quadrature with application to recurrent events and individual participant data metaanalysis. Stat Med 2014;33:3844-58

57 Perneger TV. Estimating the relative hazard by the ratio of logarithms of event-free proportions. Contemp Clin Trials 2008;29:762-6.

58 DerSimonian R, Laird N. Meta-Analysis in clinical trials. Control Clin Trials 1986;7:177-88.

59 IntHout J, loannidis JPA, Borm GF. The Hartung-Knapp-SidikJonkman method for random effects meta-analysis is straightforward and considerably outperforms the standard DerSimonian-Laird method. BMC Med Res Methodol 2014;14:1-12.

60 Higgins JPT, Thompson SG. Quantifying heterogeneity in a metaanalysis. Stat Med 2002;21:1539-58.

61 Burgess S, White IR, Resche-Rigon M, et al. Combining multiple imputation and meta-analysis with individual participant data. Stat Med 2013;32:4499-514. 
62 Burke DL, Ensor J, Riley RD. Meta-analysis using individual participant data: one-stage and two-stage approaches, and why they may differ. Stat Med 2017;36:855-75.

63 Egger M, Davey Smith G, Schneider M, et al. Bias in meta-analysis detected by a simple, graphical test. BMJ 1997;315:629-34.

64 Heffner JE, Nietert PJ, Barbieri C. Pleural fluid $\mathrm{pH}$ as a predictor of pleurodesis failure: analysis of primary data. Chest 2000;117:87-95.

65 Ahmed I, Debray TPA, Moons KGM, et al. Developing and validating risk prediction models in an individual participant data meta-analysis. BMC Med Res Methodol 2014;14:3.
66 Akaike $\mathrm{H}$. A new look at the statistical model identification. IEEE Trans Automat Contr 1974;19:716-23.

67 Hua H, Burke DL, Crowther MJ, et al. One-Stage individual participant data meta-analysis models: estimation of treatmentcovariate interactions must avoid ecological bias by separating out within-trial and across-trial information. Stat Med 2017;36:772-89.

68 Stewart LA, Tierney JF. To IPD or not to IPD? advantages and disadvantages of systematic reviews using individual patient data. Eval Health Prof 2002;25:76-97. 